KUNS-1695

hep-th/0011002

\title{
Descent Relation of Tachyon Condensation from Boundary String Field Theory
}

\author{
Sanefumi MoriYAmA ${ }^{*}$ and Shin NAKAmurA ${ }^{1,2 \dagger}$ \\ ${ }^{1}$ Department of Physics, Kyoto University, Kyoto 606-8502, Japan \\ 2 The Graduate University for Advanced Studies, Tsukuba 305-0801, Japan
}

November, 2000

\begin{abstract}
We analyze how lower-dimensional bosonic D-branes further decay, using the boundary string field theory. Especially we find that the effective tachyon potential of the lower-dimensional D-brane has the same profile as that of D25-brane.
\end{abstract}

*moriyama@gauge.scphys.kyoto-u.ac.jp

${ }^{\dagger}$ nakamura@gauge.scphys.kyoto-u.ac.jp 


\section{Introduction}

Since the discovery of D-brane [1], our understanding of string theory has been deepened.

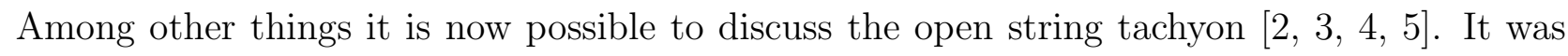
conjectured that bosonic D-brane of any dimension can decay into the closed string vacuum or lower-dimensional D-brane. Moreover the vacuum energy of the bosonic D-brane is considered to correspond to the tension of the D-brane.

Old days calculation [6] in the open string field theory [7] has been renewed to discuss the tachyon condensation [8, 9, 10, 11, 12, 13, 14, 15]. Since in these cases all scalar quantities may acquire vacuum expectation values, we can only analyze the tachyon condensation by truncating the infinite levels of string excitations. Some attempts for the exact manipulation are found in 16, 17, 18, 19.

However, recently this difficulty has been overcome [20, 21, 22]. Using another formulation called boundary string field theory (BSFT) [23, 24, 25, 26, 27] we have only to consider the tachyon field in discussing the tachyon condensation. This is because the general property of the renormalization group flow ensures that the quadratic modes of the tachyon field decouple from the other modes. Exact analysis was performed in this formulation. Both the situations that D25-brane decays into the closed string vacuum and that D25-brane decays into lowerdimensional brane are analyzed, and Sen's conjectures relating the vacuum energy and the brane tension are confirmed exactly. The derivative truncated effective action of the tachyon field obtained from BSFT is found to agree with the toy model proposed in [28, 29]. Several related works are also found in [30, 31, 32, 33, 34, 35].

In this paper we use this formulation to discuss the descent relation of tachyon condensation. We analyze, after D25-brane decays into lower-dimensional brane, how it further decays. Especially we find that the effective tachyon potential of the lower-dimensional brane has the same profiles as that of D25-brane.

In the next section, we review some results of BSFT for later necessity. After the review we proceed in Sec. 3 to analyze the descent relation using BSFT. Here our method may seem unusual from the field theoretical viewpoint. Hence in Sec. 4, we restate the results using the field theoretical analysis and furthermore justify our method used in Sec. 3. We conclude in the final section.

\section{Boundary String Field Theory}

In this section, we shall review some results of BSFT [23, 24, 25, 26, 27] which is necessary for later analysis. The BSFT is constructed [23] by identifying several contents in the Batalin- 
Vilkovisky (BV) formalism with those in string field theory. Hence we shall first shortly recall the BV formalism. The BV formalism is defined as follows. Assume that a supermanifold $\mathcal{M}$ with local coordinates $\lambda^{i}$ and fermionic closed 2-form $\omega_{i j}$ is given. If the action $S$ satisfies the master equation $\{S, S\}=0$ with the antibracket defined as $\{A, B\}=A \overleftarrow{\partial_{i}} \omega^{i j} \overrightarrow{\partial_{j}} B$, the action $S$ will necessarily have gauge symmetry. The existence of the action $S$ is equivalent to the existence of a vector field $V^{i}$ satisfying the nilpotency condition $V^{2}=0$ and the local integrability condition $d\left(i_{V} \omega\right)=0$. The two quantities $S$ and $V^{i}$ are related via $i_{V} \omega=d S$.

To construct BSFT we have to identify the contents in the BV formalism with those in string field theory. We first identify the supermanifold $\mathcal{M}$ as the space of open string fields $\left\{\mathcal{O}_{i}\right\}$ and the fermionic 2-form $\omega_{i j}$ as the two point function of the string fields $\omega_{i j}=\left\langle\mathcal{O}_{i} \mathcal{O}_{j}\right\rangle$. Here the string field $\mathcal{O}$ has ghost number one and is related to the boundary perturbation $S_{\partial \Sigma}=\int d \theta \mathcal{V}$ by the relation $\mathcal{O}=c \mathcal{V}$. Besides if the vector $V^{i}$ is identified as the BRST current vector, the derivative of the action is given as

$$
d S=\frac{1}{2} \int d \theta d \theta^{\prime}\left\langle d(c \mathcal{V})(\theta)\left\{Q_{\mathrm{BRST}}, c \mathcal{V}\right\}\left(\theta^{\prime}\right)\right\rangle_{\mathcal{V}} .
$$

It is possible to integrate this derivative as [26]

$$
S(\lambda)=Z(\lambda)\left(1+\beta^{i}(\lambda) \frac{\partial}{\partial \lambda^{i}} \log Z(\lambda)\right)
$$

with some beta functions $\beta^{i}(\lambda)$ and the partition function $Z(\lambda)$ given as

$$
Z(\lambda)=\int \mathcal{D} X \exp \left(-S_{\Sigma}(X)-S_{\partial \Sigma}(X, \lambda)\right)
$$

In the special case of the tachyon field $T$ in the quadratic profile,

$$
T=a+\sum_{i} \frac{u_{i}}{2 \alpha^{\prime}} X_{i}^{2}
$$

the BSFT action is given as [24]

$$
S\left(a, u_{i}\right)=e^{-a} \prod_{i} Z_{1}\left(u_{i}\right)\left(1+a+\sum_{i} u_{i}-\sum_{i} u_{i} \frac{\partial}{\partial u_{i}} \log Z_{1}\left(u_{i}\right)\right)
$$

where $Z_{1}(u)$ is defined as

$$
Z_{1}(u) \equiv \sqrt{u} e^{\gamma u} \Gamma(u)
$$

If we expand this action with respect to the coordinate $u$ up to the next to leading order,

$$
S\left(a, u_{i}\right)=\left(14+a+\sum_{i} u_{i}+O\left(u^{2}\right)\right) e^{-a} \prod_{i} \frac{1}{\sqrt{u_{i}}}
$$


we can rewrite the action in the field theoretical form:

$$
S=T_{25} \int d^{26} x\left(\alpha^{\prime} e^{-T}\left(\vec{\partial}_{x} T\right)^{2}+e^{-T}(T+1)\right)
$$

or

$$
S=4 e \cdot T_{25} \int d^{26} x\left(\alpha^{\prime}\left(\vec{\partial}_{x} \phi\right)^{2}-\frac{1}{4} \phi^{2} \log \phi^{2}\right)
$$

with the D25-brane tension $T_{25}$ defined as $1 /\left(2 \pi \alpha^{\prime}\right)^{13}$ in this case. (See [21, 22].) Here we have normalized the kinetic term in the usual way via the field redefinition $\log \phi^{2}=-(T+1)$. The coordinates $x^{i}$ of the target space correspond to the zero modes of the worldsheet fields $X^{i}$.

The exact BSFT action (2.5) is analyzed carefully in [21]. If we set all $u_{i}$ zero except only one direction $u_{1}=u$, we can depict the profile of the action as in Fig. 1 . Here three stationary points are found and they correspond to the perturbative vacuum of D25-brane $(a=0, u=0)$, D24-brane $(a=\infty, u=\infty)$ and the closed string vacuum where open strings condense $(a=\infty, u=0)$, respectively. When D25-brane decays into D24-brane we have to take both $a$ and $u$ to infinity under the relation

$$
a=-u+u \frac{\partial}{\partial u} \log Z_{1}(u)
$$

which is the stationary condition of $a$.

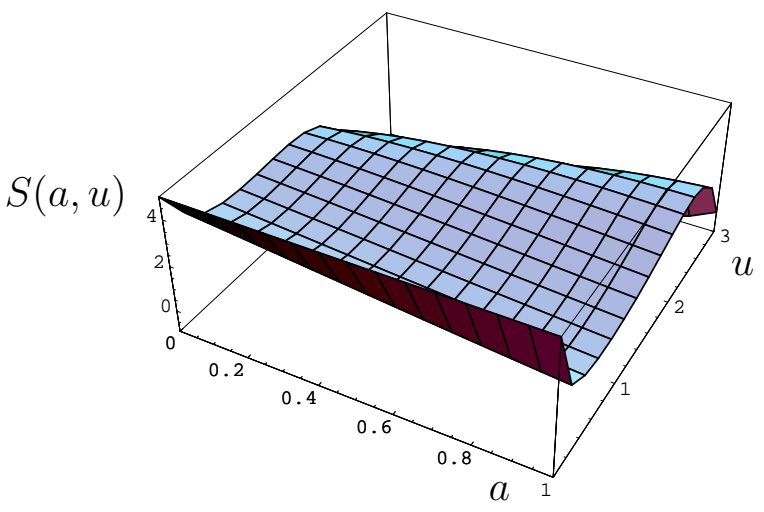

Figure 1: The profile of the BSFT action $S(a, u)$. The D24-brane is expressed as the stationary point $a, u \rightarrow \infty$ along the ridge (2.10). 


\section{Descent Relation from Boundary String Field Theory}

Here we shall analyze how lower-dimensional D-brane further decays. For simplicity we shall study the effective tachyon potential and further decays of D24-brane. Therefore we keep only two directions $T=a+\left(u X^{2}+v Y^{2}\right) / 2 \alpha^{\prime}$ for consideration: one $\left(u_{1}=u, X_{1}=X\right)$ is used when D25-brane decays into D24-brane and the other $\left(u_{2}=v, X_{2}=Y\right)$ is kept to discuss further decays. Hence the previous action $S\left(a, u_{i}\right)$ is rewritten as $S(a, u, v)$. The generalizations to other decays are straightforward.

First note that after D25-brane decays into D24-brane both the coordinates $a$ and $u$ go to infinity. However, if we would like to discuss further decay, it is necessary to identify the coordinate that plays the role of the tachyon zero mode of D24-brane, just like the coordinate $a$ in the case of D25-brane. There must be some choices. But as we shall discuss later, it should be natural to consider the section of a large constant $u=u_{*}$ and use again the coordinate $a$ for the tachyon zero mode with the origin shifted by $a_{*}$.

More precisely, we shall evaluate $S\left(a_{*}+a, u_{*}, v\right)$ in the limit $a_{*}, u_{*} \rightarrow \infty$. The double limit is taken under the relation (2.10) for $\left(a_{*}, u_{*}\right)$ since we consider the decay of D24-brane here. The calculation is straightforward if we use the asymptotic forms around $u \sim \infty$ :

$$
\begin{aligned}
\log Z_{1}(u) & \sim u \log u-u+\gamma u+\log \sqrt{2 \pi}, \\
u \frac{\partial}{\partial u} \log Z_{1}(u) & \sim u \log u+\gamma u .
\end{aligned}
$$

After a little algebra we find the following result:

$$
\lim _{a_{*}, u_{*} \rightarrow \infty} S\left(a_{*}+a, u_{*}, v\right)=\sqrt{2 \pi} e^{-a} Z_{1}(v)\left(1+a+v-v \frac{\partial}{\partial v} \log Z_{1}(v)\right) .
$$

Amazingly, this has exactly the same form as the action of the tachyon field on D25-brane except that the dimension is lowered by one and the factor $\sqrt{2 \pi}$ appears. Hence, here we have found heuristically that the profile of the tachyon potential of D24-brane is the same as that of D25-brane. Namely, the tachyon potential has self-similarity. This result makes it possible to discuss further decays of D24-brane in the same way as that of D25-brane.

We shall make a few comments here.

First our result here seems natural. D-brane of any dimension can decay into lowerdimensional D-brane or the closed string vacuum. Hence it is natural to expect that D-brane of any dimension should all have the same structure in the tachyon mode.

Secondly, as we have mentioned before our analysis, there are some choices to identify the tachyon zero mode of D24-brane. For example, though here we have fixed the coordinate $u$ to be a large constant $u_{*}$, we can alternatively fix the coordinate $a=a_{*}$ and try to regard the 
coordinate $u$ as the tachyon zero mode. This time we have to evaluate the action $S\left(a_{*}, u_{*}-u, v\right)$ in the limit $a_{*}, u_{*} \rightarrow \infty$. Although in this case we also find the self-similarity, it is not the coordinate $u$ but

$$
a_{\mathrm{eff}} \equiv u_{*} \log u_{*}-\left(u_{*}-u\right) \log \left(u_{*}-u\right)+(\gamma-1) u
$$

that plays the role of the tachyon zero mode. However, as we shall discuss in the next section, it is most natural to identify the coordinate $a$ as the tachyon zero mode as in (3.2).

\section{Field Theoretical Analysis}

In the previous section we have analyzed how the lower-dimensional D-brane further decays using the exact BSFT action. We have found that the profile of the tachyon potential is unchanged under the descent relation. However, what we have done is somewhat unusual from the field theoretical viewpoint. In the present section we would like to complement the previous analysis using the field theoretical action (2.9) 28, 29 obtained by truncating the higher derivatives. In this method we can also obtain the same results. Besides, it would shed light on our previous question: what coordinate plays the role of the tachyon zero mode of the lower-dimensional D-brane. Therefore, our analysis here justifies the previous interpretation of $S\left(a_{*}+a, u_{*}, v\right)$ as the action of the tachyon field on the lower-dimensional D-brane.

The derivative truncated action (2.9) is written as

$$
S=4 e \cdot T_{25} \int d^{25} y d x\left(\alpha^{\prime}\left(\vec{\partial}_{y} \phi\right)^{2}+\alpha^{\prime}\left(\partial_{x} \phi\right)^{2}-\frac{1}{4} \phi^{2} \log \phi^{2}\right) .
$$

As in the previous section here we also consider the effective action of the tachyon field on D24-brane for simplicity. The target space coordinate $x$ denotes the transverse direction of the D24-brane and $\vec{y}$ the longitudinal directions. The D24-brane is expressed as a classical lump solution $\bar{\phi}(x)$. To see the fluctuation on the D24-brane we have to expand the tachyon field into infinite modes around this solution as

$$
\begin{aligned}
\phi(x, \vec{y}) & =\bar{\phi}(x)+\tilde{\phi}(x, \vec{y}), \\
\tilde{\phi}(x, \vec{y}) & =\sum_{n} \xi_{n}(\vec{y}) \psi_{n}(x) .
\end{aligned}
$$

If we want to obtain the effective action for the lowest mode of the D24-brane, we have to put the expansion (4.2) with the classical solution $\bar{\phi}(x)$ and the fluctuating modes $\tilde{\phi}(x, \vec{y})$ into the original derivative truncated action (4.1) and integrate over the coordinate $x$. As shown in [29], when we calculate the effective action for the lowest mode, the effect of the higher 
excited modes does not come in. It was also found that both the profiles of the lump solution $\bar{\phi}(x)$ and the lowest mode of D24-brane $\psi_{0}(x)$ are the gaussian functions:

$$
\bar{\phi}(x)=\psi_{0}(x)=\exp \left(-\frac{1}{8 \alpha^{\prime}} x^{2}\right) .
$$

After a straightforward calculation we find the derivative truncated action (4.1) becomes

$$
S=4 e \cdot T_{25} \sqrt{4 \pi \alpha^{\prime}} \int d^{25} y\left(\alpha^{\prime}\left(\vec{\partial}_{y}\left(1+\xi_{0}(\vec{y})\right)\right)^{2}-\frac{1}{4}\left(1+\xi_{0}(\vec{y})\right)^{2} \log \frac{\left(1+\xi_{0}(\vec{y})\right)^{2}}{e}\right) .
$$

If we rewrite the lowest mode $\xi_{0}(\vec{y})$ into a new field $\phi_{\text {eff }}(\vec{y})$ as

$$
1+\xi_{0}(\vec{y}) \equiv \sqrt{e} \phi_{\mathrm{eff}}(\vec{y})
$$

we find that the derivative truncated action is unchanged except that the dimension of the field theory is lowered by one and the tension becomes $T_{25} 2 \pi \sqrt{\alpha^{\prime}} \cdot(e / \sqrt{\pi})$ which is $T_{24}$ in the interpretation of [29, 21].

Here we have repeated the analysis of the effective action of D24-brane using the field theoretical method. We have found that the result is perfectly consistent with our expectation and the result of the previous section.

Let us return back to the question raised in the previous section: why we have identified the coordinate $a$ as the tachyon zero mode with the origin shifted. To answer this question from the field theoretical analysis, first let us see how the manipulation in this section relates to the manipulation in the previous section.

In the analysis of BSFT in the previous section, we have first assumed the quadratic profile for the tachyon field (2.4) and obtained directly the integrated expression (2.5). In obtaining the action of the tachyon field on D24-brane, we have restricted ourselves in the section of a large constant $u=u_{*}$ and considered $S\left(a_{*}+a, u_{*}, v\right)$.

On the other hand, in obtaining the effective tachyon action using the field theoretical analysis in the present section, we have rewritten the field $\phi(x, \vec{y})$ as $\exp \left(-x^{2} / 8 \alpha^{\prime}\right) \times\left(1+\xi_{0}(\vec{y})\right)$ and integrated over the coordinate $x$. In other words, since both the classical lump solution $\bar{\phi}(x)$ and the lowest fluctuating mode $\psi_{0}(x)$ have the same quadratic exponent with fixed coefficient $1 / 8 \alpha^{\prime}$, it is possible to factorize the field $\phi$ as the product of the classical solution $\bar{\phi}$ in the direction $x$ and the fluctuating mode $\phi_{\text {eff }}$ in the direction $\vec{y}$ as

$$
\phi=\bar{\phi}\left[a_{*}, u_{*}, 0\right] \times \sqrt{e} \phi_{\mathrm{eff}}[a, 0, v] .
$$

Here we have defined the notation $\phi[a, u, v]$ (and their cousins $\bar{\phi}$ and $\phi_{\text {eff }}$ ) as

$$
\phi[a, u, v] \equiv \exp \left\{-\frac{1}{2}\left(a+\frac{u}{2 \alpha^{\prime}} x^{2}+\frac{v}{2 \alpha^{\prime}} \vec{y}^{2}+1\right)\right\}
$$


using the relation $\log \phi^{2}=-(T+1)$ and set $a_{*}=-1$ and $u_{*}=1 / 2$ for the derivative truncated action (4.1). Note that the right-hand-side of (4.6) is equal to $\phi\left[a_{*}+a, u_{*}, v\right]$. Hence, what we have done in this section is exactly to consider the action $S\left(a_{*}+a, u_{*}, v\right)$ in the section of $u_{*}=1 / 2$ with the origin of $a$ shifted by $a_{*}=-1$ from the viewpoint of the integrated form (2.7). Here the coordinate $a$ again corresponds to the tachyon zero mode on D24-brane. This is nothing but what we did in the previous section. In this way we have related the manipulation in this section to that in the previous section and justified the method of Sec. 3.

However, it might be dangerous to generalize the above arguments naively to the exact BSFT action. The exact BSFT action (2.5) is written not as the field theory but in the integrated form. Hence, though we know that the classical solution should be quadratic, it is difficult in principle to discuss the fluctuating modes around the classical solution and see whether the effect of the higher fluctuating modes in (4.2) comes in. Moreover, if we want to relate the two manipulations, the classical lump solution $\bar{\phi}(x)$ and the lowest fluctuating mode $\psi_{0}(x)$ should have the same gaussian profiles. Here even in the exact case of the previous section, we have optimistically assumed these properties and analyzed $S\left(a_{*}+a, u_{*}, v\right)$ in the same way as the derivative truncated case.

\section{Conclusion}

In this paper we have analyzed the descent relation of the tachyon condensation. Especially we have found that the profile of the tachyon potential of lower-dimensional D-brane is the same as that of D25-brane. Namely, the tachyon potential has self-similarity.

In discussing the effective action of the tachyon field on the lower-dimensional D-brane using BSFT in Sec. 3, we had to know how to deal with the tachyon zero mode. Here we made a non-trivial suggestion that we should shift the origin of the tachyon zero mode and consider the BSFT action $S\left(a_{*}+a, u_{*}, v\right)$ in the limit $a_{*}, u_{*} \rightarrow \infty$.

Furthermore, we repeated the analysis in the field theoretical model in Sec. 4. We found that what we did in the field theoretical model is essentially to shift the origin of the tachyon zero mode and consider the derivative truncated action $S\left(a_{*}+a, u_{*}, v\right)$ with $a_{*}=-1$ and $u_{*}=1 / 2$. This is nothing but the method used in Sec. 3. Hence, we also justified the above suggestion, using the more familiar field theoretical analysis.

Finally let us give a comment on the structure of the closed string vacuum. When we discuss the situation that D25-brane decays into the closed string vacuum, we set all $u_{i}$ zero and consider the coordinate $a$ becomes infinity. However, if we discuss the decay of D24-brane into the closed string vacuum, first we have to consider $a \rightarrow \infty$ with a large constant $u=u_{*}$ and send $u_{*} \rightarrow \infty$ afterwards. Hence, our closed string vacuum in this case seems to be 
obtained in the limit $a, u \rightarrow \infty$ and different from the original closed string vacuum $(a=\infty$, $u=0)$. This is a very delicate problem that relates to how we take the double limit. To clarify the vacuum structure at $a=\infty$ should be an interesting direction.

\section{Acknowledgment}

We would like to thank Y. Michishita, S. Shinohara and especially H. Hata for valuable discussions and comments. This work is supported in part by Grant-in-Aid for Scientific Research from Ministry of Education, Science, Sports and Culture of Japan (\#04633). One of the authors (S. M.) is supported in part by the Japan Society for the Promotion of Science under the Predoctoral Research Program.

\section{References}

[1] J. Polchinski, "Dirichlet-branes and Ramond-Ramond charges", Phys. Rev. Lett. 75 (1995) 4724, hep-th/9510017.

[2] A. Sen, "Stable non-BPS bound states of BPS D-branes", JHEP 9808 (1998) 010, hepth/9805019.

[3] A. Sen, "Tachyon condensation on the brane antibrane system", JHEP 9808 (1998) 012, hep-th/9805170.

[4] A. Sen, "SO(32) spinors of type I and other solitons on brane-antibrane pair", JHEP 9809 (1998) 023, hep-th/9808141.

[5] A. Sen, "Descent relations among bosonic D-branes", Int. J. Mod. Phys. A14 (1999) 4061, hep-th/9902105.

[6] V.A. Kostelecky and S. Samuel, "On a nonperturbative vacuum for the open bosonic string", Nucl. Phys. B336 (1990) 263.

[7] E. Witten, "Noncommutative Geometry And String Field Theory", Nucl. Phys. B268 (1986) 253.

[8] A. Sen and B. Zwiebach, "Tachyon condensation in string field theory", JHEP 0003 (2000) 002, hep-th/9912249.

[9] W. Taylor, "D-brane effective field theory from string field theory", Nucl. Phys. B585 (2000) 171, hep-th/0001201.

[10] N. Moeller and W. Taylor, "Level truncation and the tachyon in open bosonic string field theory", Nucl. Phys. B583 (2000) 105, hep-th/0002237. 
[11] J.A. Harvey and P. Kraus, "D-Branes as unstable lumps in bosonic open string field theory", JHEP 0004 (2000) 012, hep-th/0002117.

[12] R. de Mello Koch, A. Jevicki, M. Mihailescu and R. Tatar, "Lumps and p-branes in open string field theory", Phys. Lett. B482 (2000) 249, hep-th/0003031.

[13] N. Moeller, A. Sen and B. Zwiebach, "D-branes as tachyon lumps in string field theory", JHEP 0008 (2000) 039, hep-th/0005036.

[14] R. de Mello Koch and J.P. Rodrigues, "Lumps in level truncated open string field theory", hep-th/0008053.

[15] N. Moeller, "Codimension two lump solutions in string field theory and tachyonic theories", hep-th/0008101.

[16] A. Sen, "Universality of the tachyon potential", JHEP 9912 (1999) 027, hep-th/9911116.

[17] L. Rastelli and B. Zwiebach, "Tachyon potentials, star products and universality", hepth/0006240.

[18] V.A. Kostelecky and R. Potting, "Analytical construction of a nonperturbative vacuum for the open bosonic string", hep-th/0008252.

[19] H. Hata and S. Shinohara, "BRST invariance of the non-perturbative vacuum in bosonic open string field theory", JHEP 0009 (2000) 035, hep-th/0009105.

[20] A.A. Gerasimov and S.L. Shatashvili, "On exact tachyon potential in open string field theory", hep-th/0009103.

[21] D. Kutasov, M. Marino and G. Moore, "Some exact results on tachyon condensation in string field theory", hep-th/0009148.

[22] D. Ghoshal and A. Sen, "Normalization of the background independent open string field theory action", hep-th/0009191.

[23] E. Witten, "On background independent open string field theory", Phys. Rev. D46 (1992) 5467, hep-th/9208027.

[24] E. Witten, "Some computations in background independent off-shell string theory", Phys. Rev. D47 (1993) 3405, hep-th/9210065.

[25] K. Li and E. Witten, "Role of short distance behavior in off-shell open string field theory", Phys. Rev. D48 (1993) 853, hep-th/9303067. 
[26] S.L. Shatashvili, "Comment on the background independent open string theory", Phys. Lett. B311 (1993) 83, hep-th/9303143.

[27] S.L. Shatashvili, "On the problems with background independence in string theory", hep-th/9311177.

[28] B. Zwiebach, "A Solvable Toy Model for Tachyon Condensation in String Field Theory", JHEP 0009 (2000) 028, hep-th/0008227.

[29] J.A. Minahan and B. Zwiebach, "Field theory models for tachyon and gauge field string dynamics", JHEP 0009 (2000) 029, hep-th/0008231.

[30] J.A. Minahan and B. Zwiebach, "Effective Tachyon Dynamics in Superstring Theory", hep-th/0009246.

[31] L. Cornalba, "Tachyon Condensation in Large Magnetic Fields with Background Independent String Field Theory", hep-th/0010021.

[32] K. Okuyama, "Noncommutative Tachyon from Background Independent Open String Field Theory", hep-th/0010028.

[33] D. Kutasov, M. Marino and G. Moore, "Remarks on Tachyon Condensation in Superstring Field Theory", hep-th/0010108.

[34] O. Andreev, "Some Computations of Partition Functions and Tachyon Potentials in Background Independent Off-Shell String Theory", hep-th/0010218.

[35] S. Dasgupta and T. Dasgupta, "Renormalization Group Analysis of Tachyon Condensation", hep-th/0010247. 